\title{
Poliovirus surveillance by examining sewage specimens. Quantitative recovery of virus after introduction into sewerage at remote upstream location
}

\author{
T. HOVI ${ }^{1 *}$, M. STENVIK ${ }^{1}$, H. PARTANEN ${ }^{1}$ AND A. KANGAS ${ }^{2}$ \\ ${ }^{1}$ Enterovirus Laboratory, WHO Collaborating Centre for Reference and Research on Poliomyelitis, National \\ Public Health Institute (KTL), Helsinki, Finland \\ ${ }^{2}$ Helsinki Water, City of Helsinki, Finland
}

(Accepted 13 March 2001)

\section{SUMMARY}

In order to assess the feasibility of environmental poliovirus surveillance, known amounts of poliovirus type 1, strain Sabin, were flushed into the sewage network of Helsinki. Grab specimens collected at a remote downstream location and concentrated about a 100-fold revealed infectious poliovirus on four successive days in all three separate experiments. As for concentration, a simple two-phase separation method was found to be at least as useful as a several-fold more resource-demanding polyethylene glycol (PEG) precipitation method.

Recovery of the introduced virus was remarkably high (more than $10 \%$ ). Using the current system, it might be possible to detect poliovirus circulation in a population of 700000 people by examining a single $400 \mathrm{ml}$ sewage specimen, if 1 out of 10000 inhabitants were excreting the virus. It is concluded that environmental surveillance is a sensitive approach to monitor silent poliovirus circulation in populations served by a sewage network.

\section{INTRODUCTION}

In the natural course of poliovirus infection excretion of poliovirus in the stools continues for a period of several weeks. Search for poliovirus in sewage or in other waste waters has been used for assessment of the extent of the epidemic spread of poliovirus and to approximate the proportion of infected individuals in the source population [1-9]. It has been suggested that environmental surveillance could be used as one approach to assess elimination of poliovirus circulation in a given human population, and to demonstrate the final success of poliovirus eradication. Evaluation of the potential benefits of environmental surveillance under developing-country conditions is in progress [10]. However, the sampling principles and virus detection methods used so far have been rather variable and often non-quantitative,

\footnotetext{
* Author for correspondence: Dr Tapani Hovi, Enterovirus Laboratory, National Public Health Institute, Mannerheimintie 166, FIN-00300 Helsinki, Finland.
}

and their feasibility for use in the WHO polio laboratory network has not been established.

Since the last outbreak of poliomyelitis in Finland in 1984-5 [5], the sewage of the Helsinki region has been regularly monitored for polioviruses on a biweekly basis. A relatively small volume of raw sewage $(400 \mathrm{ml})$ from the inlet of a major sewage treatment plant has been collected, concentrated 50-100-fold and tested for infectious poliovirus in cell culture [5], in recent years using a poliovirus-selective recombinant murine cell line expressing the human poliovirus receptor on the cell surface $[11,12]$. No polioviruses have been found in the sewage since 1985. Because the inactivated poliovirus vaccine (IPV), rather than the live attenuated oral vaccine (OPV) is used in Finland in regular immunizations, OPVderived poliovirus strains are not expected to occur in the environment, unlike in most other countries.

Some years ago, the sewage treatment system in the Helsinki region was reorganized, and the single major treatment plant is now processing wastewaters cover- 
ing a population of about 740000 . In order to assess the population sensitivity of our approach for poliovirus surveillance under these new conditions, we introduced known amounts of poliovirus Sabin type 1 in the sewage network, and studied kinetics of its appearance at the regular sampling site. We also compared two sample concentration methods that are used to improve the sample sensitivity of poliovirus detection.

\section{METHODS}

\section{Source population and the sewage network}

A single sewage treatment plant collects sewage in an area of about $350 \mathrm{~km}^{2}$, and serves a population of about 740000 people living in Helsinki and the neighbouring municipalities. Practically all inhabitants and premises in the region are connected to the same sewage network. The distance of the major sewage plant from our institute is about $20 \mathrm{~km}$, including two pumping stations. The mean volume of daily sewage flow at this sewage plant was about 200000000 litres during the study period.

\section{Preparation of virus stocks}

The attenuated poliovirus type 1 Sabin strain (PV1S) was the reference strain for OPV potency control from the National Institute for Biological Standards and Control (NIBSC), Potters Bar, UK. In our laboratory it was passaged twice in HEp2 (Cincinnati subline) cells before use as an inoculum (diluted 1 in 100) to infect three sets of 20 Roux bottles of HEp2 cells each. After full cytopathic effect (CPE) in the cultures, they were frozen and thawed three times to release the virus, and kept frozen at $-20{ }^{\circ} \mathrm{C}$ until use as test stocks. Virus concentration of the stock virus preparations was determined by end-point titration in tube cultures of HEp2. The three lots had titres of $10^{7 \cdot 5}, 10^{7.8}$ and $10^{7.5} \mathrm{CCID}_{50} / \mathrm{ml}$, respectively.

\section{Sample collection and concentration}

For the purposes of routine sewage monitoring at the plant, a volumetrically regulated automatic sampling apparatus withdrew $200 \mathrm{ml}$ samples from the inlet pipe of the sewage plant after each $5000 \mathrm{~m}^{3}$, and pooled samples collected during each successive $24 \mathrm{~h}$ period into a container kept at $4{ }^{\circ} \mathrm{C}$. For this study, aliquots of these pooled samples were used. One litre of each mixture was transported to our institute, and processed immediately. Two different methods were used for concentration.

Our in-house method based on the principle of twophase separation [13], was slightly modified from the standard procedure described by Pöyry et al. [5]. Briefly, $400 \mathrm{ml}$ of raw sewage was centrifuged for $10 \mathrm{~min}$ at $1500 \mathrm{~g}$. The pellet was stored and the supernatant was concentrated with the dextranpolyethylene glycol (PEG)-mixture (Dextran T500 from Pharmacia Fine Chemicals, Uppsala, Sweden, and PEG 3000 from Lääketukku Oy, Helsinki, Finland). After overnight incubation at $4{ }^{\circ} \mathrm{C}$ in a separation funnel, a standard volume of $4 \mathrm{ml}$ was harvested combining the small lower phase and the hazy interphase. The pellet from the first centrifugation was suspended in this concentrate and the suspension was extracted with $1 \mathrm{ml}$ of chloroform by shaking vigorously, followed by clarification of the water phase by table-top centrifugation as above.

The alternative method, PEG-precipitation, was carried out using a procedure slightly modified from that described by Shieh et al. [14]. Briefly, $400 \mathrm{ml}$ of raw sewage was first spun for $10 \mathrm{~min}$ at $10000 \mathrm{~g}$ in a Sorvall High Speed centrifuge. The pellet (I) was kept cold, and the supernatant mixed thoroughly with $32 \mathrm{~g}$ of PEG 6000 (Fluka Chemie AG, Buchs, Switzerland). After overnight at $4{ }^{\circ} \mathrm{C}$, the precipitate was collected by centrifuging for $30 \mathrm{~min}$ at $10000 \mathrm{~g}$. The supernatant was discarded, and the pellet was dissolved in $4 \mathrm{ml}$ of buffer. The original pellet I was resuspended in the solution and the suspension was extracted with chloroform as above.

\section{Cell cultures used in virus detection}

Two cell lines were used for inoculation. L20B is a recombinant murine cell line permanently expressing the human poliovirus receptor (PVR). It is susceptible to poliovirus but non-permissive to most other human enteric viruses [11]. RD cells are derived from human rhabdomyosarcoma tumour tissue and are susceptible to most human enteroviruses, excluding the coxsackie $\mathrm{B}$ viruses. Our stocks of the cell lines were derived from the NIBSC (L20B) and the National Institute for Public Health and Environment (RIVM), The Netherlands (RD), respectively. After preparing a local working cell bank from an early passage, the cells were passaged maximally 15 times before restarting the cultures from the working cell bank. Putative contamination by bacteria, fungi and myco- 
Table 1. Detection of enteroviruses in sewage samples

\begin{tabular}{llllll}
\hline \hline \multirow{2}{*}{ Expt } & & \multicolumn{4}{l}{ Virus serotype isolated from sewage } \\
\cline { 3 - 6 } & Cell line & Day 1* & Day 2 & Day 3 & Day 4 \\
\hline 1 & L20B & PV1 & PV1 & Negat & PV1 \\
2 & RD & EV6+EV11 & EV6 & EV6 & EV11 \\
2 & L20B & PV1 & PV1 & PV1 & PV1 \\
3 & RD & EV6+EV11 & PV1+EV6 & EV6+EV11 & PV1+EV6 \\
& L20B & PV1 & PV1 & PV1 & PV1 \\
& RD & EV6+EV11 & EV11 & EV6+EV11 & EV11 \\
\hline \hline
\end{tabular}

* Aliquots from sewage samples were concentrated 100-fold and tested in the two cell lines. PV1, poliovirus type 1/Sabin. EV, echovirus.

plasmas was monitored regularly. The culture medium was the minimal essential medium (MEM) supplemented with $10 \%$ foetal calf serum $(2 \%$ in the maintenance medium), and penicillin and streptomycin.

\section{Assays for cytopathic viruses and virus identification}

Aliquots of the chloroform-extracts of the sewage concentrates $(0.5 \mathrm{ml})$ were inoculated in duplicate 25 $\mathrm{cm}^{2}$ flasks of the two cell lines. After $60 \mathrm{~min}$ at $36^{\circ} \mathrm{C}$, unattached inoculum was removed and the cultures were re-fed with $5 \mathrm{ml}$ of maintenance medium. Virus replication was monitored by daily microscopy, and cultures showing definite CPE (usually greater than $50 \%$ ) were harvested and frozen for virus identification. If necessary, a blind passage was made after about 10 days of inoculation. Virus identification was performed with standard techniques using intersecting LBM-type pools of neutralizing enterovirus antisera, followed by confirmation by monotypic antisera. For titration of poliovirus in the extracts, $100 \mu 1$ aliquots of tenfold dilutions were inoculated in monolayers of L20B cells in microtitre plates, 5 wells per dilution. The results were scored on days 5-6.

\section{RESULTS AND DISCUSSION}

\section{Partial delay of poliovirus migration justifies grab sampling}

In order to investigate the fate of poliovirus in a city sewage network, we introduced 3-6 $\times 10^{10} \mathrm{CCID}_{50}$ of PV1S in the sewerage of Helsinki by a single flush at a toilet at the Institute. Volumetrically adjusted samples of sewage corresponding to four subsequent $24 \mathrm{~h}$ periods were collected from the inlet of the relevant sewage treatment plant. Three separate experiments were carried out, at a one-month interval each. Samples of the raw sewage were concentrated about 100-fold, and chloroform extracts of the concentrates were assayed for infectious virus using poliovirus susceptible cell lines. Poliovirus was detected at the sewage plant already during the first $24 \mathrm{~h}$ of introduction and persisted in the specimens until day 4 . All but of the 12 specimens collected in the three experiments tested positive for PV (Table 1). Routine sewage specimens collected before, after and in between the experiments were negative for PV (data not shown).

These results indicate that migration of a bolus sample of poliovirus introduced in a city sewage network is efficiently desynchronized with the front of the bolus rapidly moving downstream but with a significant proportion of the specimen showing a delayed pattern of migration in the sewerage. This behaviour is most likely due to the complex structure of a sewage network including multiple turns in the pipes, varying flow rates in different locations, and the two pumping stations to enable 'uphill' migration. The resulting mechanical mixing is also likely to blend natural excreta from infected individuals with the main bulk of sewage contents, thus allowing representative sampling at the main sewage plant. The observed temporal spread of the bolus sample over several days during migration supports the view that the grab-type sampling method can yield, under the conditions used, sewage specimens that are representative of the source population. Furthermore, because of this pattern of bolus spreading over several days, the stepwise collection extended to $24 \mathrm{~h}$ is not necessary, but a single grab would most likely give the same level of accuracy.

The alternative sampling principle would be to use 
an adsorbing trap immersed in the sewage stream for extended periods, and then elute or extract the virus from the adsorbing material. Although reportedly useful and relatively sensitive [7, 15] the traps are likely to be easily blocked by suspended solids in the sewage, and the denominator for quantitative assessments is difficult to define.

\section{Quantitative recovery of introduced poliovirus from sewage network}

Poliovirus was detected at the sampling site during four successive days after introduction into the sewage system. The concentration usually peaked on days $1-2$ (Table 2). Hence, the introduced virus was diluted in at least four daily volumes of the sewage flow, jointly corresponding to about 800 million litres. This would result in a theoretical maximum mean amount of $15-30 \mathrm{CCID}_{50} \mathrm{PV}$ in an assayed $400 \mathrm{ml}$ volume. Since all but one of the specimens examined were positive for poliovirus, some of them even at a dilution of 1 in 10 , the proportion of recoverable $\mathrm{PV}$ at the sampling site appeared to be definitely above $10 \%$. It is not possible to assess the recovered proportion more accurately as the poliovirus concentration in the specimens was so low.

Overall, the recovered proportion of the introduced poliovirus was surprisingly high, suggesting that this approach can be effectively used to monitor poliovirus excretion at a single sampling site for a relatively large human population. After all, it is unlikely that significant proportions of introduced poliovirus would be adsorbed to the surfaces in the sewage network as these must be already saturated by other adsorbing materials. Likewise, the main stream of the sewage containing the suspended poliovirus is likely to bypass any dead ends along the migration route. There is no evidence suggesting that the regular sewage in the Helsinki region would contain poliovirus-inactivating compounds in significant concentrations. Since all parts of the sewage network in Helsinki are deeply underground, elevated temperatures are not likely to occur during the migration. These factors may explain the observed high-percentage recovery.

\section{Methodological aspects of sample concentration and virus detection}

If the proportion of PV excreting individuals is high in the source population, there is no need to concentrate the sewage specimen [4, 9]. For the documentation of the likely absence of poliovirus circulation in a given population, however, it is necessary to concentrate the virus in a raw sewage sample. Several different principles have been successfully used for this purpose. Adsorption to cationic membranes (reviewed in [16 and 17]) or to glass wool [18], followed by elution, ultrafiltration of chloroform treated samples [8], precipitation by acidification or PEG [14] are among the frequently used principles of PV concentration from sewage. In our present study, all 12 specimens collected were concentrated in parallel with the two methods adjusting the nominal concentration power to 100 -fold. In our experiments the PEG precipitation method yielded slightly less polioviruses than the in-house two-phase separation (Table 2). The difference is unlikely to be of practical importance as in other experiments, carried out on sewage samples spiked with known amounts of four separate wild type PV strains, similar recoveries were obtained from the two concentration methods (data not shown). Undiluted inocula of the two-phaseconcentrates appeared toxic in microwell cultures of L20B cells more often than the PEG-concentrates. This may have been based on the relatively large inoculum volume per cell monolayer area, as no toxicity was seen with the $20 \mathrm{~cm}^{2}$ flasks. In our regular sewage monitoring, similar $0.5 \mathrm{ml}$ concentrates are inoculated into $25 \mathrm{~cm}^{2}$ monolayers. In our experience over several years, toxicity of sewage specimens is a rarely occurring phenomenon in flask cultures of L20B cells.

As regards the necessary equipment and required hands-on time at the different steps of the concentration procedure, there was an important difference between the two methods compared. While the most fancy apparatus needed for the two-phase separation is a simple shaker, and even it can most likely be replaced by a magnetic stirrer, the PEG precipitation method requires several large volume and/or high speed centrifugations. In a standard high-speed centrifuge, only two specimens of this volume range can be processed simultaneously. In our laboratory, the hands-on time for processing two samples simultaneously was several-fold greater in the PEG precipitation method than in the two-phase separation method.

The selective power of the L20B cells was once again demonstrated by the fact that all but one of the specimens yielded a positive poliovirus, and only poliovirus, in these cells, whereas echovirus type 6 or 11 or both, but no polioviruses, were isolated in RD 
Table 2. Recovery of poliovirus from sewage samples

\begin{tabular}{|c|c|c|c|c|c|c|}
\hline \multirow[b]{4}{*}{ Sample numbers } & \multicolumn{6}{|c|}{ Test system and isolation result } \\
\hline & \multicolumn{3}{|c|}{ Two-phase separation } & \multicolumn{3}{|c|}{ PEG-precipitation } \\
\hline & \multirow[b]{2}{*}{ Flask } & \multicolumn{2}{|c|}{ Microwell } & \multirow[b]{2}{*}{ Flask } & \multicolumn{2}{|c|}{ Microwell } \\
\hline & & $10^{0}$ & $10^{-1}$ & & $10^{0}$ & $10^{-1}$ \\
\hline $1-1$ & + & Toxic & $+1 / 5$ & - & - & - \\
\hline $1-2$ & + & Toxic & $+2 / 5$ & + & $+1 / 5$ & - \\
\hline $1-3$ & - & - & - & - & - & - \\
\hline $1-4$ & + & - & - & + & - & - \\
\hline $2-1$ & + & - & - & + & $+2 / 5$ & $+1 / 5$ \\
\hline $2-2$ & + & $+4 / 5$ & $+1 / 5$ & + & $+2 / 5$ & - \\
\hline $2-3$ & + & Toxic & - & + & - & - \\
\hline $2-4$ & + & - & - & - & - & - \\
\hline $3-1$ & + & Toxic & $+1 / 5$ & + & $+4 / 5$ & - \\
\hline $3-2$ & + & $+5 / 5$ & - & + & - & - \\
\hline $3-3$ & - & NT & NT & + & - & - \\
\hline $3-4$ & + & $+5 / 5$ & - & + & - & - \\
\hline
\end{tabular}

$400 \mathrm{ml}$ samples of raw sewage were concentrated to $4 \mathrm{ml}$ and $0.5 \mathrm{ml}$ aliquots were inoculated into L20B cell monolayers in $25 \mathrm{~cm}^{2}$ flasks or, as such and as a $10^{-1}$ dilution, into microwell cultures, 5 wells per dilution. + and - , positive and negative isolation of poliovirus type 1 , respectively. $+x / y$ stands for the number of wells with CPE over the number of wells inoculated.

cells in most cases from the PV-containing specimens (Table 1). Although the PVR-expressing mouse Lcells are not absolutely selective for poliovirus [11, 12] this result is in line with the previous reports, and with wide experience within the WHO Polio Laboratory Network [19] indicating that these cells are extremely useful in the analysis of specimens where a non-polio enterovirus would mask the presence of poliovirus in the standard cell lines.

\section{Conclusions}

These experiments confirmed that a simple grab sampling of raw sewage may be used in environmental surveillance of poliovirus circulation in a given population served by a defined sewage network. This is based on the apparently efficient blending and mixing of any introduced material with the bulk of sewage contents. Since sewage usually contains abundant non-polio enteroviruses, a selective cell line such as the L20B used in our experiments, is necessary to avoid masking of small amounts of poliovirus in the samples. In countries where OPV is used in general immunisations, additional selection means have to be used to guarantee that OPV-derived viruses would not mask small amounts of wild-type poliovirus in the samples [9]. Concentration of grab samples is required to increase the detection sensitivity. In our hands, the in-house two-phase separation method appeared as effective as but much less resource-demanding than the PEG-precipitation method. One has to remember, however, that the concentration power of the PEG precipitation method could be significantly increased without much extra working load while this is not true for our in-house method. Whatever method is being used, it is important to assess the actual performance of the method as locally executed, for instance, using sewage samples spiked with known amounts of poliovirus. Ranta and co-workers [20] have presented formulae that can be used to approximate the influence of changes in sample sensitivity on virus detection probability at the population level.

In the current experiments, a remarkably large proportion of input poliovirus was recovered from the sewage samples collected during the four successive days. The input amount of virus, $3-5 \times 10^{10} \mathrm{CCID}_{50}$, could be estimated to represent an output of about 400-750 persons (taking a mean concentration of $10^{5}$ CCID $_{50}$ per gram of stool, and a daily amount of $200 \mathrm{~g}$ of stool, would result in a mean 4-day contribution of $8 \times 10^{7} \mathrm{CCID}_{50}$ per an infected child of $20 \mathrm{~kg}$ ). Because already small fractions of the concentrates yielded poliovirus, even a significantly smaller amount of poliovirus excreting individuals 
among the 740000 people in the region (i.e. perhaps fewer than 1 out of 10000) might become detected by examining a single sewage specimen. It has been shown that repeated sampling will rapidly increase the population sensitivity [20]. Therefore, under conditions where the recovery rate of input poliovirus cannot be assessed, environmental surveillance with repeated sampling should be continued for extended periods to maximize the detection probability.

\section{REFERENCES}

1. Zdrazilek J, Sromova H, Hoffmanova V. Comparison of poliovirus detection in sewage and stool samples; a study in a creche in the third week after vaccination. Int J Epidemiol 1977; 6: 169-72.

2. Sellwood J, Dadswell JV, Slade JS. Viruses in sewage as an indicator of their presence in the community. J Hyg 1981; 86: 217-25.

3. Lapinleimu K. Elimination of poliomyelitis in Finland. Rev Infect Dis 1984; 6 (Suppl 2): 457-60.

4. Hovi T, Cantell K, Huovilainen A, et al. Outbreak of poliomyelitis in Finland: widespread circulation of antigenically altered poliovirus type 3 in a vaccinated population. Lancet 1986; 1: 1427-32.

5. Pöyry T, Stenvik M, Hovi T. Viruses in sewage waters during and after a poliomyelitis outbreak and subsequent nationwide oral poliovirus vaccination campaign in Finland. Appl Environm Microbiol 1988; 54: 371-4.

6. Böttiger M, Herrstrom E. Isolation of polioviruses from sewage and their characteristics: experience over two decades in Sweden. Scand J Infect Dis 1992; 24: $151-5$.

7. Tambini G, Andrus JK, Marques E, Boshell J, Pallansch M, de Quadros CA, Kew OM. Direct detection of wild poliovirus circulation by stool surveys of healthy children and analysis of community wastewater. J Infect Dis 1993; 168: 1510-4.

8. van der Avoort HG, Reimerink JH, Ras A, Mulders $\mathrm{MN}$, van Loon AM. Isolation of epidemic poliovirus from sewage during the 1992-3 type outbreak in The Netherlands. Epidemiol Infect 1995; 114: 481-91.
9. Manor Y, Handsher R, Halmut T, et al. Detection of poliovirus circulation by environmental surveillance in the absence of clinical cases in Israel and the Palestinian authority. J Clin Microbiol 1999; 37: 1670-5.

10. World Health Organization. Fourth informal consultation of the polio laboratory network, Geneva, 1-2 October 1998. WHO, Geneva, 1999; WHO/ V\&B99.05:11-2.

11. Pipkin PA, Wood DJ, Racaniello VR, Minor PD. Characterization of $\mathrm{L}$ cells expressing the human poliovirus receptor for specific detection of polioviruses in vitro. J Virol Meth 1993; 41: 333-40.

12. Hovi T, Stenvik M. Selective isolation of poliovirus in recombinant murine cell line expressing the human poliovirus receptor gene. J Clin Microbiol 1994; 32: 1366-8.

13. Albertson P. Two-phase separation of viruses. In: Maramorosch K, Koprowski H, eds. Methods of virology. Academic Press Inc., New York, 1967: 303-21.

14. Shieh Y-SC, Wait D, Tai L, Sobsey MD. Methods to remove inibitors in sewage and other fecal wastes for enterovirus detection by the polymerase chain reaction. J Virol Meth 1995; 54: 51-66.

15. World Health Organization. Environmental surveillance of wild poliovirus circulation in Europe. WHO/ EURO, Copenhagen, 1992. EUR/ICP/EPI 019/ 8000B:3.

16. Rao VC, Metcalf TG, Melnick JL. Human viruses in sediments, sludges and soils. Bull WHO 1986; 64: 1-14.

17. Metcalf TG, Melnick JL, Estes MK. Environmental virology: from detection of virus in sewage and water by isolation to identification by molecular biology - a trip over 50 years. Ann Rev Microbial 1995; 49: 461-87.

18. Wood DJ, Hull B. L20B cells simplify culture of polioviruses from clinical specimens. J Med Virol 1999; 58: $188-92$.

19. Grabow WOK. Waterborne diseases: update of water quality assessment and control. Water SA 1996; 22: 193-202.

20. Ranta J, Hovi T, Arjas E. Poliovirus surveillance by examining sewage water specimens. Studies on detection probability using simulation models. Risk Anal. In press. 